\title{
Indoor Fuzzy Self-Localization using Fuzzy Segments
}

\author{
D. Herrero-Pérez and H. Martínez-Barberá
}

\begin{abstract}
The research presented in this paper approaches the issue of indoor localization using the fuzzy logic framework for modeling and dealing with the uncertainty of the position measurements. Fuzzy logic presents properties that make it a suitable tool to represent and manage the different factors that affect the measures. This framework allows representing the perceptions, including their associated uncertainty, using fuzzy sets and making use of the tools provided by the framework to manage and operate them. This work uses the fuzzy segment theory to maintain a coherent local representation around the robot using multi-sensor fusion based on fuzzy logic, and uses these fuzzy segments to feed a fuzzy self-localization method, which is able to deal with the ambiguity in the global localization problem.
\end{abstract}

Index Terms-Fuzzy Logic, Indoor Localization, Multi-sensor Fusion, Intelligent Systems.

\section{INTRODUCTION}

$\mathbf{T}$ HE indoor localization problem consists on estimating the robot's location using absolute (reference-based systems) and relative (also called dead-reckoning) position measurements [3], which give the situation of the environment around the robot and the feedback about its driving actions respectively. Basically, it consists on answering the question Where am I? [16] from the robot's point of view given: a representation of the world where the robot is navigating and sensor information. Localization methods are usually distinguished according to the type of problem they tackle [13]; in particular, i) tracking or local techniques, and ii) global techniques. Commonly, local techniques present advantages in accuracy and efficiency, while global techniques are much more robust [14].

What makes this problem difficult is the presence of uncertainty in the measurements because it usually results hard to represent noisy measures. These measures are usually stated by giving a range of values that are likely to enclose the real value. From the probabilistic point of view, this range is usually designated by repeating the measure under the same conditions enough times for modeling the uncertainty using a probabilistic function. In other words, probabilistic functions should be experimentally calculated which implies reproducing the measure under the same conditions. However, sometimes it is not possible to repeat the measure or reproduce the factors that influence this. Techniques based on fuzzy logic only require an approximate sensor model [6] of the measures. Moreover, position measurements may be affected by different types

D. Herrero-Pérez is with University of Murcia. E-mail: dherrero@dif.um.es

H. Martínez-Barberá is with University of Murcia. E-mail: humberto@um.es of uncertainty, including vagueness, imprecision, ambiguity, unreliability, and random noise. Furthermore, it is probable that more than one factor influences the measurements, which are not usually independents. Consequently, the formalism used to represent the uncertainty should be able to represent all of these types of uncertainty and to account for the differences between them.

Fuzzy logic presents properties that makes it suitable to represent and handle the different facets of the uncertainty in the measurements [20]; fuzzy sets are used to represent the measures and their associated uncertainty, while fuzzy logic provides the framework to combine these measures because it provides power tools to match the degree of similarity between the measures represented by fuzzy sets. In addition, these techniques are applicable in domains where traditional methods fail or not satisfy many assumptions. For instance, when the sensor model is unknown methods based on frequencies are not suitable, while fuzzy logic based methods are. In fact, these techniques have already shown their advantages in other domains, such as fuzzy control and modeling [18], [17], [24], [22], [23], both in the Mobile Robotics framework.

Therefore, instead of probabilities many authors represent the location uncertainty using fuzzy sets; in particular, to address localization and mapping problems. For instance, the world has been modeled using fuzzy metric features, like fuzzy segments [9], or using a fuzzy grid map [18]. In addition, many works make use of a fuzzy model of the environment to locate the robot on this representation of the world, like [10] where the localization problem consists on find out the correspondence between the fuzzy local map built by the robot and the fuzzy model of the environment. In [19] a fuzzy landmark-based localization method is proposed where extracted clues are matched against an approximate map of the environment to obtain a location's estimation, instead of finding out the correspondences, this method treats each observation as a source of partial location information combining relative and absolute position measurements using a fuzzy aggregation operator. Besides, the method proposed in [6] combines ideas from the Markov localization approach proposed in [5] with ideas from the fuzzy landmark-based approach technique proposed in [19]. These fuzzy techniques have also been used for addressing the Simultaneous Localization and Mapping (SLAM) problem [12], [11] by extracting segments from range data which form the local map, this is then matched to the global map, the matching gives a new position to the robot, and then the local map is either added to the global map, if the area the local map occupies was not explored before, or the global map is updated with the local map. 


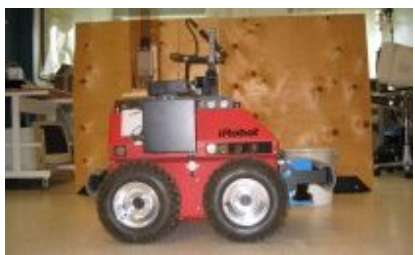

(a)

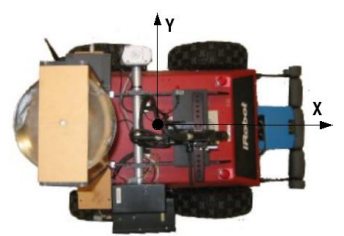

(b)
Fig. 1. (a) ATRV Jr robot manufactured by iRobot, which is equipped with a laser rangefinder and skid steering. (b) Local reference system.

This work deals with the uncertainty of the position measurements to locate a robot in an indoor known-environment using fuzzy techniques. The robot is equipped with a laser rangefinder as exteroceptive sensory system and skid steering. The laser rangefinder provides dense and accurate range measures, high sampling rate, high angular resolution and good range distance, while skid steering results in unreliable odometry. The absolute measurements are represented by the fuzzy segments proposed by Gasós [11], where uncertainty on the real location of a line-segment is represented by a fuzzy set, i.e., the degree of membership to the fuzzy set represents the uncertainty location. In other words, the local perception around the robot at time $t$ is represented as a set of linesegments with its uncertainty represented by fuzzy sets, and fuzzy logic is used to manage and fuse the detections during the navigation taking into account the different factors and sources of uncertainty. This allows maintaining a coherent representation of the environment around the robot during the navigation. This representation is used to feed the fuzzy self-localization technique proposed in [6] and extended in [15], where the robot's belief is modeled as a distribution on a $2 \frac{1}{2} \mathrm{D}$ possibility grid. This localization method based on fuzzy logic allows us to represent and track multiple possible locations where the robot might be. In addition, it only requires an approximate model of the sensor system and a qualitative estimation of the robot's displacement. Thus, the method presented is able to maintain a coherent representation of the environment surrounding the robot using imprecise and unreliable driving measures.

The paper is structured as follows: Section II is devoted to obtain the position measurements to estimate the robot's pose, both sensing and acting; in particular, the detection and fusion of fuzzy segments during the navigation using the laser rangefinder to maintain a coherent representation of the environment around the robot taking into account the different sources of uncertainty that affect to the measurements for the sensing and the estimation of the distance traveled by the robot using a very unreliable steering. Section III is focused on the fuzzy self-localization method to estimate the robot's location using the sensed fuzzy segments. Section IV presents the experimental validation of the method proposed and finally, conclusions are presented in Section V.

\section{Position MEAsurements}

This section presents the platform and sensory system, and also the techniques based on fuzzy logic to obtain the position

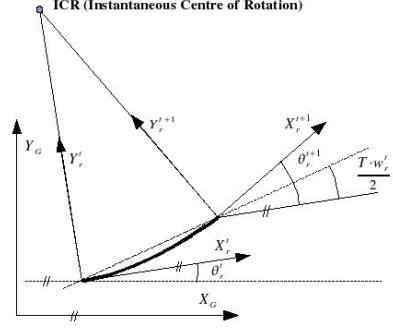

(a)

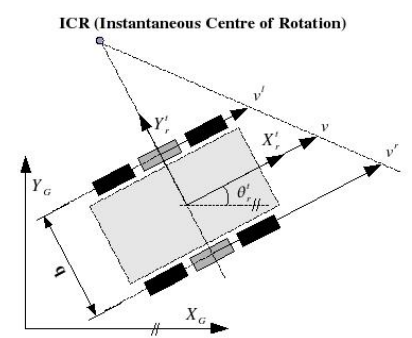

(b)
Fig. 2. (a) Assumptions of the kinematics model and (b) differential drive model to estimate the distance the robot has traveled.

measurements representing the different factors that affect the uncertainty of these measures. These position measurements, both relative and absolute, are used to feed the self-localization method also based on fuzzy logic.

The platform is an ATRV Jr manufactured by iRobot, which relies on skid steering, i.e., two wheels on each side are linked. In order to rotate, each wheel pair rotates at a different speed, which inevitably gives rise to a sliding whenever a rotation is performed making the odometry quite unreliable. The robot is further equipped with devices that provide range measures to obstacles; in particular, 17 sonar mostly pointing forward and to the sides and a SICK LMS laser rangefinder pointing forward giving 180 degrees view, although we only use the scanner in the experiments of this work. The whole system is shown in Fig. 1, including the local reference system to calculate the local perceptions and relative displacements.

The techniques used to obtain the relative and absolute position measurements are outlined below.

\section{A. Position measurements from actions}

The wheeled robot is equipped with odometry sensors that measure the amount each wheel has rotated, which are used to estimate the relative distance and rotation the robot has traveled. The odometry measurements consist on one value of the distance the robot has traveled and one value of the angle the robot has rotated.

Normally, mobile robots use quite simple mathematical models to describe their instantaneous motion capabilities. In addition, these kinematics models make many assumptions to simplify the calculation of the relative displacement. Our kinematics model considers trajectories with fixed curvature during the measure of the displacement, i.e., we are considering a trajectory of fixed curvature during the sampling, which is usually valid if the sampling rate is sufficiently high. Fig. 2(a) shows the local coordinates of a displacement during the period $T$. Following these assumptions, the new location of the robot given the estimation of the velocities $\left\langle v_{r}, w_{r}\right\rangle$ using the values of the odometers is calculated using the Equation (1). 


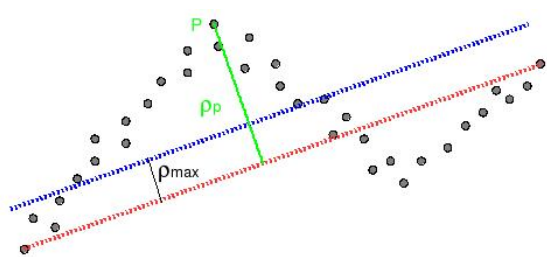

(a)

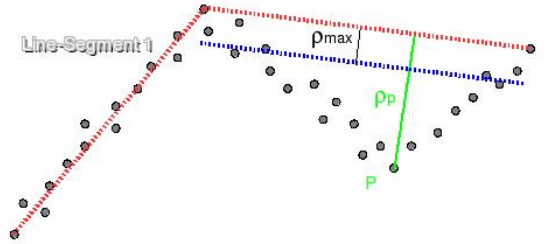

(b)

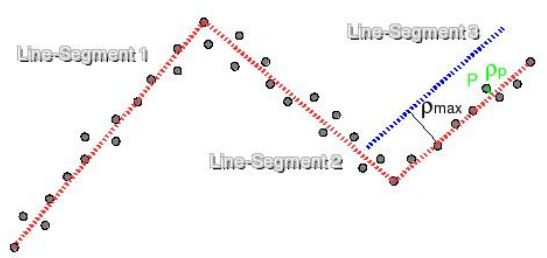

(c)

Fig. 3. Example of Iterative-End-Point-Fit algorithm: (a) initial splitting process given $N$ points, (b) non-stop and (c) stop criterion.

$$
\left[\begin{array}{l}
x_{r}^{t+1} \\
y_{r}^{t+1} \\
\theta_{r}^{t+1}
\end{array}\right]=\left[\begin{array}{c}
x_{r}^{t} \\
y_{r}^{t} \\
\theta_{r}^{t}
\end{array}\right]+T \cdot\left[\begin{array}{cc}
\cos \left(\theta_{r}^{t}+\frac{T \cdot w_{r}^{t}}{2}\right) & 0 \\
\sin \left(\theta_{r}^{t}+\frac{T \cdot w_{r}^{t}}{2}\right) & 0 \\
0 & 1
\end{array}\right] \cdot\left[\begin{array}{c}
v_{r}^{t} \\
w_{r}^{t}
\end{array}\right]
$$

The estimation of the velocities $\left\langle v_{r}, w_{r}\right\rangle$ is calculated using the angle rotated by each wheel and the physical characteristics of the platform. In our case, we are using the differential drive model that operates with the velocity of each pair of wheels. This model is derived assuming the balance conditions shown in Fig. 2(b), where the velocities of each pair of wheels induce a rotation around the instantaneous center of rotation I. Equations (2) and (3) estimate the linear and angular velocities respectively, being $v^{l}$ and $v^{r}$ the velocities of the left and right wheels respectively.

$$
\begin{aligned}
v_{r} & =\frac{v^{r}+v^{l}}{2} \\
w_{r} & =\frac{v^{r}-v^{l}}{b}
\end{aligned}
$$

However, this model is only an approximation assuming each pair of wheels rotates the same angle and have the same diameter. In practice, wheels usually have slight different diameters and the physical characteristics of the platform are not perfect. In addition, slight changes in the velocities of these linked pairs perform unbalanced conditions that induce slippages. Skid steering inevitably gives rise to slippages whenever a rotation is performed. Thus, the uncertainty in position measures from actions of this robot is high and unpredictable, and the techniques used to deal with this uncertainty should represent and take into account these factors.

\section{B. Position measurements from perceptions}

In order to obtain the absolute position location, we are using a laser rangefinder as main exteroceptive sensor, which provides dense and accurate range measures, high sampling rate, high angular resolution and good range distance. Laser rangefinders are based on the measure of the time of flight of a pulse of optical energy emitted against an object which reflects the signal to the receptor, i.e., they provide the distance to the closest object in the direction the pulse is emitted. In the case of scanner lasers, they provide a set of measures in different orientations (scan) at time $t$.
In order to address the localization problem, it is paramount to being able to find out the correspondence between measurements to match: i) the measures sensed at different times and thus filtering out false detections, and also ii) the measures with the a priori map provided to the robot in the localization problem. The most common matching techniques in mobile robotics are point-based and feature-based matching. Pointbased methods deal with raw data directly, they normally result extremely complex and inefficient, and therefore they can not be used directly in high level tasks, at least with an acceptable performance. Feature-based methods transform the raw data into high level features, which can be efficiently used in matching problems. Logically, algorithms based on features are expected to be more efficient than point-based algorithms.

This work aims to locate the robot on a metric map known a priori, which is composed of geometric primitives; in particular, line-segments representing the walls and the obstacles of an indoor environment. Hence, we should use the more suitable technique to extract line-segments from the readings of the laser. Line extraction quality is of paramount importance for line-based localization because wrong feature extraction can lead the system to divergence. An evaluation of the more popular line extraction methods using range sensors is performed by Siegwart in [21], where the algorithms are evaluated in terms of complexity, speed, correctness and precision, being Split-and-Merge algorithm the preferred method for the localization problem using an a priori map, and the best choice for real-time applications by its clearly higher speed. Therefore, we are using a variant of the generic Split and Merge method to obtain the line-segments from the laser scans; in particular, a simplified version namely Iterative-EndPoint-Fit algorithm [7], which has been used in many recently researches [27], [4]. The advantages of this line extraction method are: i) it only needs two values to tune the method, while Split-and-Merge algorithm needs three, ii) the algorithm only splits sets and iii) it does not need to fit the grouped data to check if the set has to be split again. This method is outlined below.

The Iterative-End-Point-Fit algorithm evaluates the whole data set through a line connecting the first and the last points of the scanning. This line is not the fitted-line of the set, but it is only a line-segment which has as end-points the first and last readings of the set to evaluate. Instead of the three parameters that we have to state in the Split-and-Merge algorithm, we only have to state two parameters to tune this variant; in particular, the minimum number of points per line-segment $N_{\min }$ and maximum distance to the hypothetical fitted line $\rho_{\max }$. The 


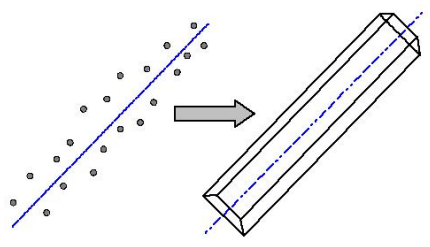

(a)

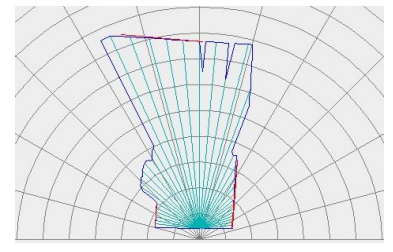

(b)
Fig. 4. (a) Representation of the uncertainty in a line-segment fitted by a least square method using fuzzy sets (from [8]) and (b) an example of the fuzzy segments generated by a scan of the laser rangefinder.

other parameter in the generic Split-and-Merge algorithm is the number of points $N$ to begin the splitting process, being the total number of points in the initial set in this variant. The algorithm works as follows:

Step 1: Draw a line-segment between first and last point of the set $s$.

Step 2: Detect the point $P$ with maximum perpendicular distance $\rho_{P}$ to the line-segment.

Step 3: If $\rho_{P}$ is higher than the threshold $\rho_{\max }$, split $s$ at $P$ into $s_{1}$ and $s_{2}$ and go to Step 2 for both sets.

Stop: When all sets, candidates to line-segments, satisfy the Step 3 and they contain at least $N_{\min }$ points.

Fig. 3 shows an example of the Iterative-End-Point-Fit recursive method. Note this method does not provide a set of line-segments, it only returns set of readings which are candidate to be fitted by a line. Hence, after grouping the data we have to use a fitting method to generate the line-segments.

In order to fit the readings into line-segments, the typical linear regression using least-squares methods is used. Leastsquares methods minimize the sum of the squares of the distances between the resulting line and the data of the set, but these distances depends on the formula used to fit the line. We are using the orthogonal regression or eigenvector line fitting because it uses the normal line equation to minimize the normal distance from the data of the set to the resulting line. This line equation is able to represent all possible lines, including vertical and horizontal.

The resulting line-segments are defined as a tuple $B=\{$ $\left.p_{\rho}, p_{\varphi},\left(x_{i}, y_{i}\right),\left(x_{f}, y_{f}\right), N\right\}$, where $p_{\varphi}$ and $p_{\rho}$ are the line parameters in normal form, $\left(x_{i}, y_{i}\right)$ and $\left(x_{f}, y_{f}\right)$ are the endpoints and $N$ is the number of readings that generates the line-segment. The endpoints are calculated by the projection of the bounds of the set over the fitted line.

In addition to the line-segments surrounding the robot at certain time, the information about the uncertainty of the sensing position measurements is of paramount importance to address properly the self-localization problem. In this work, we are using the fuzzy segment theory proposed by Gasós [8] to represent and deal with the uncertainty on the real location of the objects. This approach represents the uncertainty on the real location of the objects using fuzzy sets, where the degree of membership to the fuzzy set represents the uncertainty in the location and fuzzy logic framework is used to match the degree of similarity between objects represented by fuzzy sets. In other words, fuzzy logic provides the framework to manage formally the uncertainty of the observations represented by

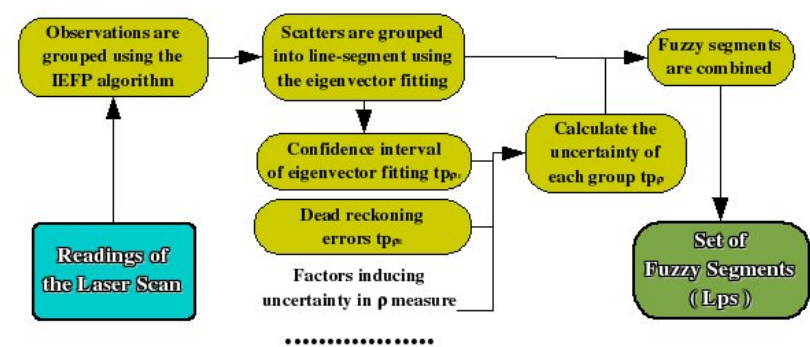

Fig. 5. Overall flowchart to obtain the coherent set of fuzzy segments surrounding the robot using the readings of a laser rangefinder.

\section{fuzzy sets.}

Following [8], perceptions are expressed as fuzzy segments, i.e., the uncertainty of the line-segment parameter $p_{\rho}$ is represented using a trapezoidal fuzzy set $t p_{\rho}$. A fuzzy segment is defined as a tuple $F S=\left(p_{\varphi}, p_{\rho}, t p_{\rho},\left(x_{i}, y_{i}\right),\left(x_{j}, y_{j}\right), N\right)$, where $t p_{\rho}$ is the trapezoidal fuzzy set representing the uncertainty in $p_{\rho}$. The uncertainty on the object's location is influenced by different factors, which usually depend on the sensory system, the platform and the processing techniques. In our case, these factors are the uncertainty of: the measure of each reading, the fitting or regression using least squares methods, and possible dead reckoning errors. The uncertainty of the two first factors is known or can be estimated, while we can not model the dead reckoning errors because skid steering gives rise to unpredictable errors. Thus, we have to state a bound for this kind of errors based on practical experience. Fig. 4(a) shows the way the uncertainty of the fitting of scatter readings is represented by a fuzzy set, which is obtained considering the confidence intervals of the least square method used by the regression method. Considering the different factors that influence in the uncertainty of the parameter $p_{\rho}$ and assuming the factors are independent, $t p_{\rho}$ is defined as the addition of the representation of all factors or uncertainties that influence the parameter $p_{\rho}$, that is, $t p_{\rho}=t p_{\rho}^{1} \oplus t p_{\rho}^{2} \oplus \ldots \oplus t p_{\rho}^{n}$.

When the environment around the robot is represented by a set of fuzzy segments generated by the laser readings at different times, the fuzzy logic framework is used to fuse and propagate the uncertainty of these perceptions. Finally, the object boundaries perceived by the robot are represented by a vector containing these fuzzy segments $M=\left\{F S_{1}, F S_{2}, \ldots, F S_{m}\right\}$, which include an estimation of the uncertainty in the detections. This vector, namely local perceptual state (LPS), is used for locating the robot in further processing. Fig. 4(b) shows an example of a set of fuzzy segments generated by many laser rangefinder scans. The blue perimeter represents the scan contour, range measures obtained by the sub-sampling method are depicted using cyan beams and red segments show the resulting set of fuzzy segments. Note the uncertainty in the $\rho$ dimension, width of segments, is quite small because the laser provides quite good distance estimations.

Fig. 5 shows the overall flowchart to obtain this representation of the environment surrounding the robot given the readings of the laser rangefinder. The process is as follows. The input is the set of readings provided by the laser scan. 


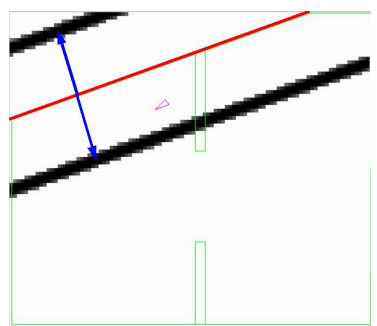

(a)

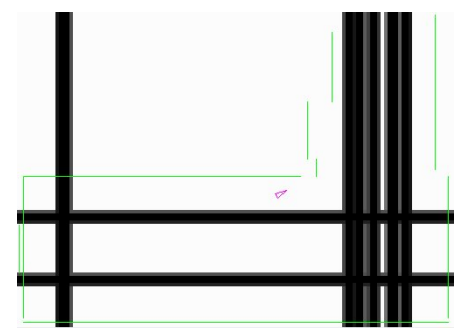

(b)
Fig. 6. Belief induced by the observation of an (a) unique and (b) non-unique feature.

First, these readings are grouped into sets which satisfy many linear conditions using the Iterative-End-Point-Fit algorithm, and then the line-segments are generated from the scatter points using a least square method. Then, the fuzzy set that estimates the uncertainty of the detection is calculated by the addition, following the independent assumption between them, of the fuzzy sets that represent the different factors that affect the observation. The resulting set of fuzzy segments is then merged with the previous representation of the environment to obtain a coherent representation around the robot and to remove repetitious information. Finally, the output is a set of fuzzy segments that represent object boundaries surrounding the robot.

\section{FUZZY SELF-LOCALIZATION}

This section describes the method based on a fuzzy grid that is used to locate the robot in an indoor known environment. The method uses the set of fuzzy segments and the estimation of the distance traveled, sensing and acting respectively, to estimate the robot's location on the given metric map. This fuzzy self-localization method was proposed by Buschka in [6] and extended in [15] to deal with the ambiguity without addressing the data association problem.

\section{Uncertainty Representation}

Because location information may be affected by different types of uncertainty, such as vagueness, imprecision, ambiguity, unreliability, and random noise, the formalism used to represent the location information should be able to represent all of these types of uncertainty and account for the differences between them. Fuzzy logic techniques are suitable in this respect [20], thus location information of an object is represented by a fuzzy subset $\mu$ of the set $X$ of all possible locations [25], [26]. For any $x \in X$, the value of $\mu(x)$ is read as the degree of possibility that the object is located at $x$ given the available information.

Following [6], the fuzzy locations are represented in a discretized format in a position grid: a tessellation of the space in which each cell is associated with a number in $[0,1]$ representing the degree of possibility that the object is in that cell. For performance reasons, a $2 \frac{1}{2} \mathrm{D}$ possibility grid is used to represent the robot's belief about its own pose, that is, its $(x, y)$ position plus its orientation $\theta$. This simplification allows to represent multiple hypotheses about different positions, but

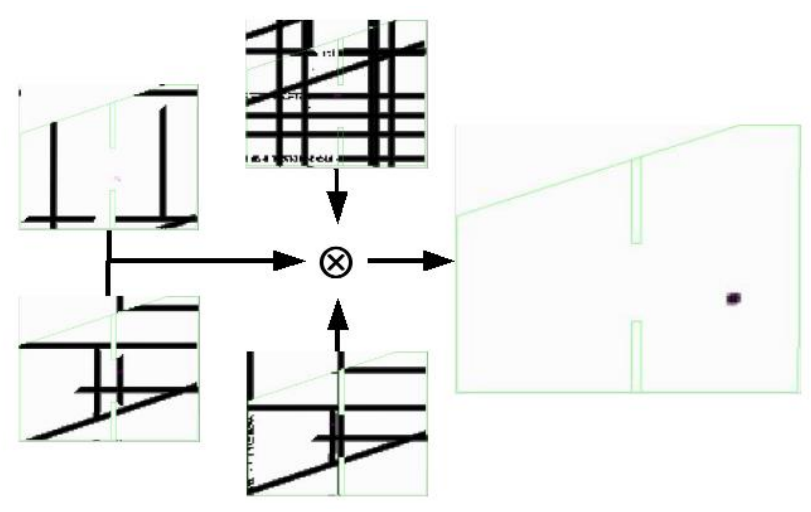

Fig. 7. Example of the fusion of many robot's beliefs induced by non-unique observations.

only one orientation hypothesis on a given $(x, y)$ position, which is acceptable in this domain.

Suppose that the robot senses a given feature at time $t$, the sensed range and bearing to the feature are represented by a vector $\vec{r}$. It knowing the position of the feature in the a priori map given to the robot, this observation induces a belief about its own position in the environment. Since there is uncertainty in the observations, this belief is affected by the uncertainty. All facets of uncertainty are represented using fuzzy locations. For every type of feature, the belief induced at time $t$ by an observation $\vec{r}$ is represented by a possibility distribution $S_{t}(x, y, \theta \mid \vec{r})$ that gives, for any pose $(x, y, \theta)$, the degree of possibility that the robot is at $(x, y, \theta)$ given the observation $\vec{r}$. This distribution constitutes our sensor model for that specific feature.

The shape of the $S_{t}(x, y, \theta \mid \vec{r})$ distribution depends on the type of feature. In the case of unique observations, this distribution is a rectangle parallel to the sensed feature, being $|\vec{r}|$ the distance in the $(x, y)$ plane, blurred according to the amount of uncertainty in the estimation of the range. In the case of multiple possible observations, the sensor model is the union of the distributions induced by all possible observations. Fig. 6(a) shows the example of the belief induced by an unique observation (red feature) at a certain distance (blue arrows) which is observable from both sides. The belief has the shape of two parallel rectangles to the observation with a trapezoidal section corresponding to the uncertainty of the fuzzy segment, i.e., the width is the support of the trapezoidal fuzzy set used by the fuzzy segment. Fig. 6(b) shows the example of the belief induced by a non-unique detection which is only observable from one side. The induced belief is composed by the union of the beliefs of all possible unique distributions, which avoids deal with the data association problem, although this approach probably delays the convergence of the localization method.

\section{Self-Localization}

The robot's belief about its own pose is represented by a distribution $G_{t}$ on a $2 \frac{1}{2} \mathrm{D}$ possibility grid. This representation allows us to represent, and track, multiple possible positions where the robot might be. When the robot is first placed on the environment, $G_{0}$ is set to 1 everywhere to represent total 
ignorance. This belief is then updated according to the typical predict-observe-update cycle of recursive state estimators as follows.

Predict: When the robot moves, the belief state $G_{t-1}$ is updated to $G_{t}$ using a model of the robot's motion. This model performs a translation and rotation of the $G_{t-1}$ distribution according to the amount of motion, followed by a uniform blurring to account for uncertainty in the estimate of the actual motion.

Observe: The observation of a feature at time $t$ is converted to a possibility distribution $S_{t}$ on the $2 \frac{1}{2}$ grid using the sensor model discussed above. For each pose $(x, y, \theta)$, this distribution measures the degree of possibility that the robot is at that pose given an observation.

Update: The possibility distribution $S_{t}$ generated by each observation at time $t$ is used to update the predicted belief state $G_{t}$ using a fuzzy intersection operator. The resulting distribution is then normalized.

If the robot needs to know the most likely position estimate at time $t$, it does so by computing the center of gravity $(\mathrm{CoG})$ of the distribution $G_{t}$. A reliability value for this estimate is also computed, based on the area of the region of $G_{t}$ with highest possibility and on the minimum bias in the grid cells. Because the center of gravity given multiple possibilities in the distribution provides a wrong estimation, outside of all the possible locations in the world, this reliability value is used to decide when the robot has obtained a right estimation of its own location. This is useful, for instance, to decide to engage in an active re-localization behavior.

In practice, the predict phase is performed using tools from fuzzy image processing, like fuzzy mathematical morphology, to translate, rotate and blur the possibility distribution in the grid [1], [2]. The intuition behind this is to see the fuzzy position grid as a gray-scale image.

For the update phase, the position grid is updated by performing pointwise intersection of the current state $G_{t}$ with the observation possibility distribution $S_{t}(\cdot \mid r)$ at each cell $(x, y)$ of the position grid. For each cell, this intersection is performed by intersecting the trapezoid in that cell with the corresponding trapezoid generated for that cell by the observation. This process is repeated for all available observations. Intersection between trapezoids, however, is not necessarily a trapezoid. For this reason, in our implementation we actually compute the outer trapezoidal envelope of the intersection. This is a conservative approximation, in that it may over-estimate the uncertainty but it does not incur the risk of ruling out true possibilities.

There are many choices for the intersection operator used in the update phase, depending on the independence assumptions that we can make about the items being combined. In our case, since the observations are independent, we use the product operator which reinforces the effect of consonant observations. Fig. 7 shows the result of the intersection between the beliefs induced by a set of fuzzy segments detected at time $t$. Note the result is an unique possible location, although this representation is able to handle more than one location in the environment.

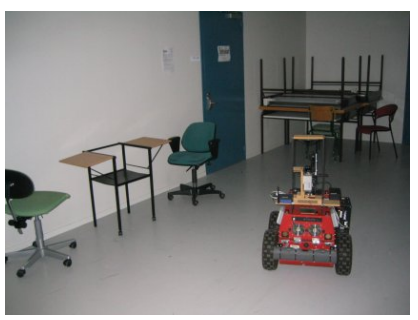

(a)

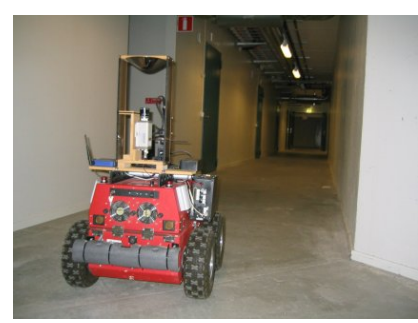

(b)
Fig. 8. Experimental set-up of the Atrv Jr robot navigating through a nonmodified environment where there are corridor, doors, radiators and furniture.

This self-localization technique has nice computational properties. Updating, translating, blurring and computing the center of gravity $(\mathrm{CoG})$ of the fuzzy grid are all linear in the number of cells.

\section{EXPERIMENTAL RESULTS}

This section presents the experiments to validate the techniques based on fuzzy logic proposed in this work. First, the experimental set-up is described, then the results of experiment performed in quite different environments are presented, and finally these results are discussed. In order to evaluate the results, the ground truth is manually estimated in strategic locations during the navigation of the robot.

\section{Experimental set-up}

The experiments using the wheeled robot are performed in two different environments: an office-like environment and the basement of a building, which is a relative large environment of approximately $15 \times 43$ meters. Fig. 8 shows that there are long corridors, radiators, doors, and office furniture like chairs and tables in the environments, which are not modified to perform the experiments. The robot is manually steered during both experiments using a joystick with the operator walking behind the robot, most of the time outside the working area. While the robot is manually driven around the known environment, the laser rangefinder and odometry readings are collected to estimate the robot's location during the experiment. The environments are static, i.e., nothing is in motion during the experiment runs, except the robot.

The purpose of these experiments is to check if the perception system is able to maintain a coherent representation of the environment surrounding the robot and provide properly information to locate the robot in the known environment, filtering out the fuzzy segments that do not satisfy the conditions to be considered as walls because the a priori known map is only composed by line-segments representing the walls of the world. In addition, these experiments aim to test if the self-localization method is able to solve the global localization problem, and then track the robot's location during the navigation. The main disadvantage of methods based on grids is the computational cost in large environments because the cell resolution limits the accuracy, and a trade off between cell size and computational cost must be found. 


\section{Experiment in an office-like environment}

The dimension of the office-like environment is approximately $12.5 \times 9.1$ meters. We are using a cell resolution of 10 centimeters, which is experimentally determined as trade-off between accuracy and computation cost for the selflocalization method. This resolution generates a fuzzy grid of $125 \times 91$ cells.

In order to evaluate the quality of the estimation, the laser rangefinder readings are used to obtain the perpendicular distance to the sensed walls, which is enough information to triangulate and obtain the ground truth knowing the match between laser readings and the walls. The ground truth is manually calculated in strategic locations of the experiment to obtain the position and heading errors, and also check if the estimation of the uncertainty is correct.

The localization method is initialized with a belief distributed along the whole environment, that is, the robot does not know its own location at the beginning. Then, the robot starts collecting range measurements. As soon as a feature is sensed or an action is performed, it is incorporated into the localization process. The resulting beliefs and the features sensed are shown in the sequence of the Fig. 9. This figure also shows the map given to the robot, which consists on a set of line-segments representing the walls of the office, the real and estimated trajectories of the experiment, which are obtained by hand and defuzzificating the fuzzy robot's belief using the center of gravity operator respectively, and the bounding boxes representing the uncertainty of the robot's position in each dimension during the navigation.

We have noticed that the fuzzy robot's belief takes the form of a unique possible location only using the information provided by the set of fuzzy segments sensed in the first step of the experiment. Thus, the localization method only needs the first three fuzzy segments, depicted in the second figure of the sequence, to obtain the fuzzy robot's belief that provides the robot's location. The real trajectory, which is manually calculated, and the estimated trajectory using the fuzzy selflocalization method are shown in Fig. 9(a) when the method provides a right estimation, i.e., when the reliability value that estimates the quality of the estimation indicates high reliability values. Note that the localization method is able to track the robot's location using the sensing and acting position measurements during the rest of the experiment. In addition, we can observe that the real location of the robot (ground truth) is always inside the bounding box during the whole experiment. This bounding box defines an area centered in the estimated robot's location, which is obtained by the center of gravity of the fuzzy grid, where it is the highest possibility that the robot is inside. Logically, this area considers the dispersion of the fuzzy grid.

The position and orientation errors are shown in Fig. 10. These errors are calculated when the self-localization method estimates high reliability values, i.e., once the localization has converged. We have noticed that the position error shows many peaks, which are due to the motion and its uncertainty model, which induces uncertainty in the robot's belief in all directions. In other words, because the robot's motion is very

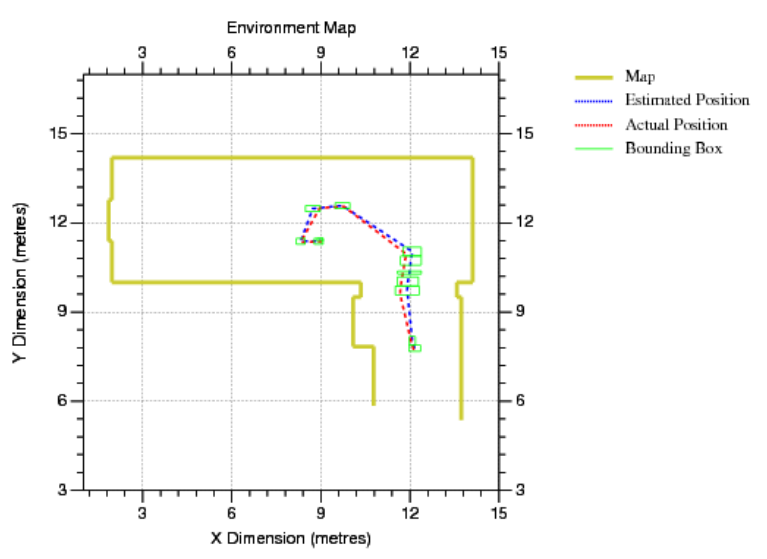

(a)
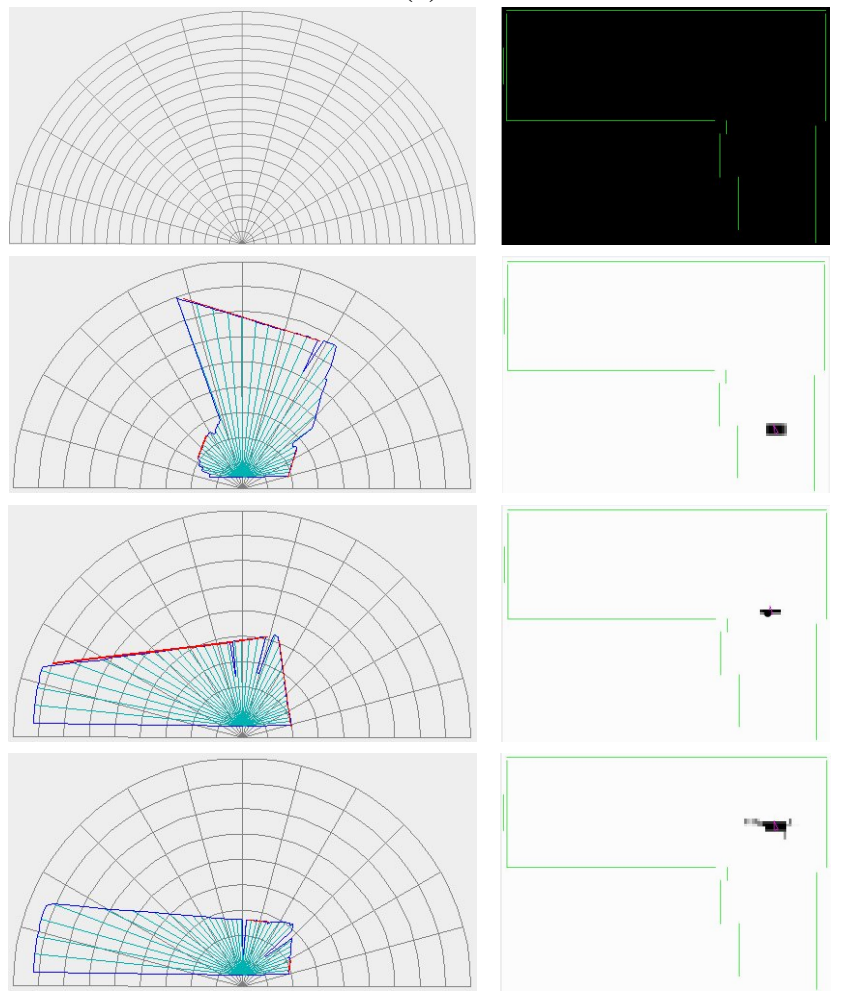

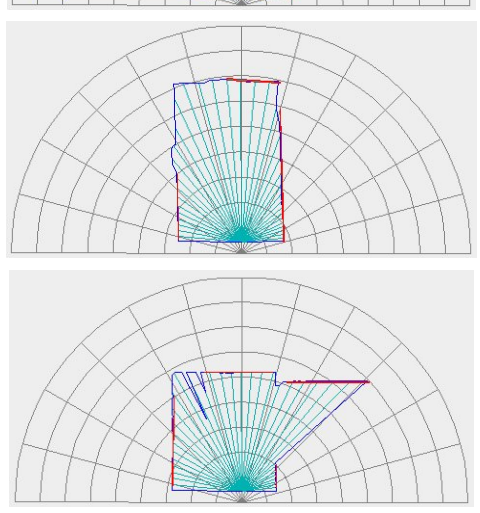

(b)

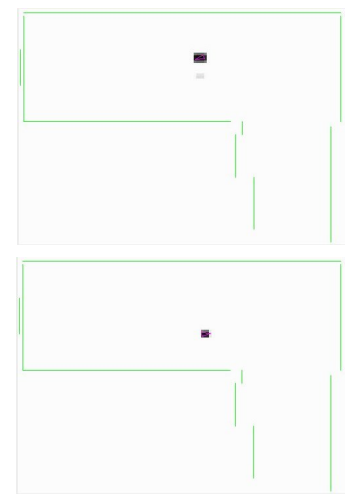

(c)
Fig. 9. Experiment in an office-like environment. (a) Map of the office, real and estimated trajectories and bounding boxes that bounds the errors in the estimation. Sequence of the experiment, both (b) fuzzy segments and (c) robot's belief are shown at different times. 


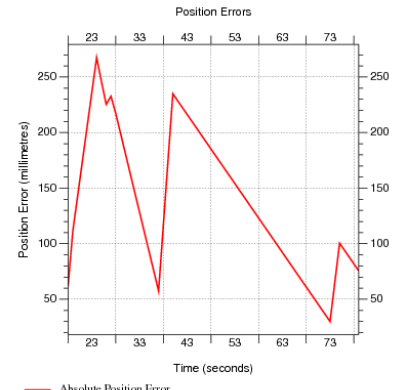

(a)

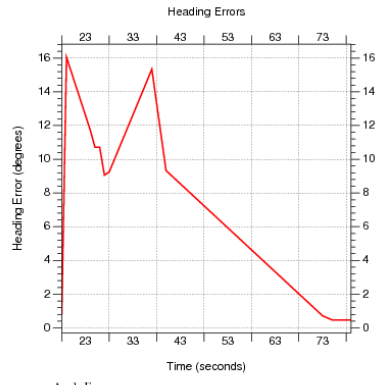

(b)
Fig. 10. (a) Position and (b) heading errors of the experiment in an office-like environment.

unreliable and we are using a very rough motion model, the robot's belief increases a lot and if there is not enough absolute information to reduce this belief again, position error is increased. Anyway, the position error is less than $30 \mathrm{~cm}$ along the whole experiment and sometimes is less than $5 \mathrm{~cm}$, while the orientation error is less than 17 degrees along the whole experiment. Hence, we can state that the fuzzy selflocalization method is able to track the robot's location when the global localization problem is solved.

The computational cost of this method depends of the cell resolution and the dimensions of the environment. We have evaluated the timing of this experiment to demonstrate that it satisfies the real time constraints for this environment providing good position estimations. Using a Pentium M at $1,73 \mathrm{GHz}$ with $512 \mathrm{MB}$ of RAM memory; the acting operation, i.e., translation, rotation and blur of the fuzzy robot's belief consumes 9 milliseconds for this environment, while the sensing operation consumes 3 milliseconds for each observation. Considering the detection of 5 fuzzy segments in average, the localization method consumes 18 milliseconds, which allows processing the measures provided by the laser at acquisition rate.

\section{Experiment in a relative large environment}

As previously mentioned, the relative large environment is a basement with long corridors which measures approximately $15.3 \times 42.6$ meters. The cell resolution is also 10 centimeters, which generates a fuzzy grid of $125 \times 91$ cells.

The ground truth is also manually calculated in strategic locations of the experiment to obtain the position and heading errors, and also check if the estimation of the uncertainty is correct. The processing to obtain this ground truth is the method described above.

The localization method is also initialized with a belief distributed along the whole environment, that is, the robot does not know its own location in the beginning. The resulting beliefs and the sensed features are shown in the sequence of the Fig. 11. This figure also shows the map given to the robot, the real and estimated trajectories of the experiment and the bounding boxes representing the uncertainty of the robot's position in each dimension during the navigation.

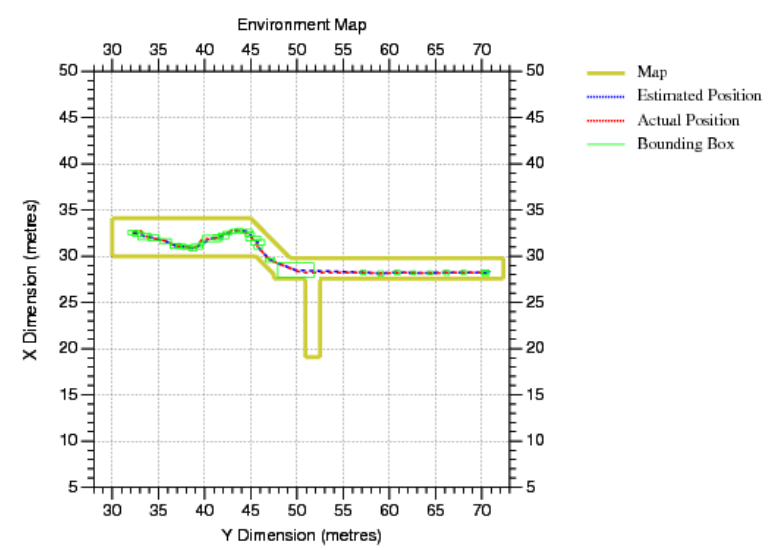

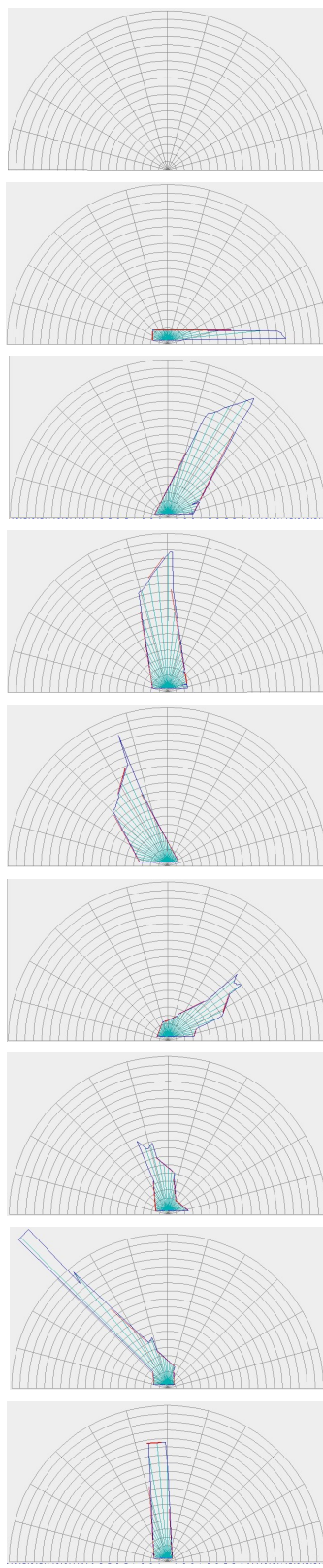

(a)
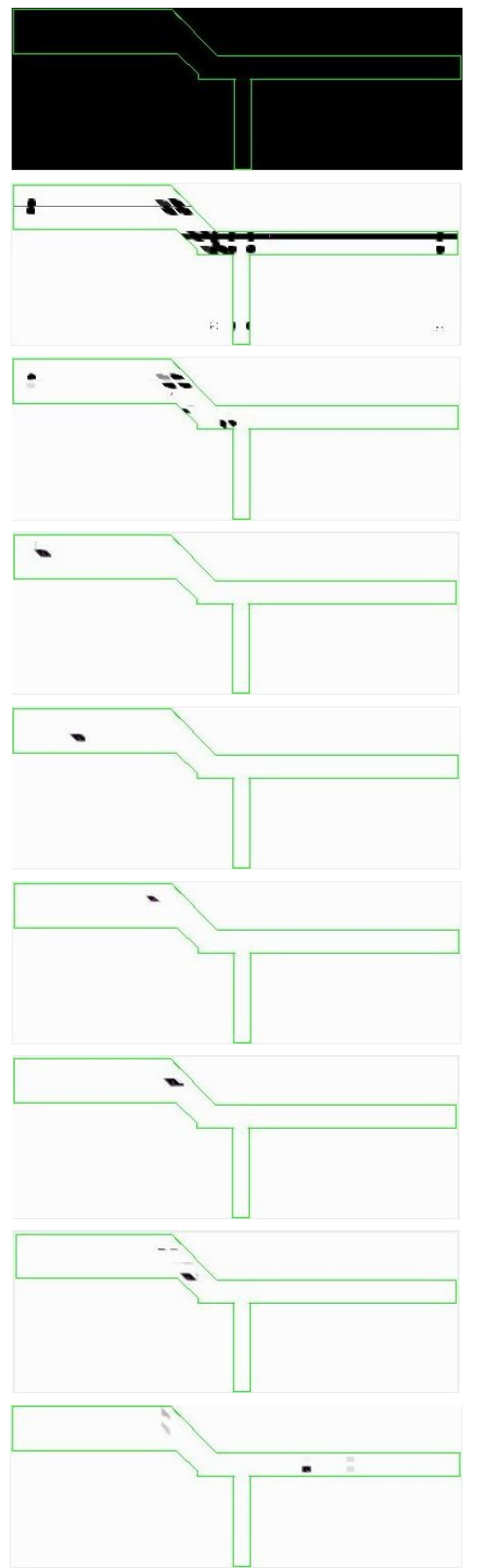

(b)
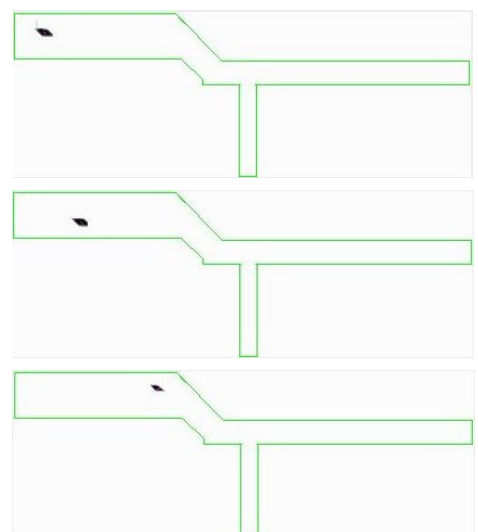

Fig. 11. Experiment in an relative large basement. (a) Map of the basement, real and estimated trajectories and bounding boxes that bounds the errors in the estimation. Sequence of the experiment, both (b) fuzzy segments and (c) robot's belief are shown at different times. 


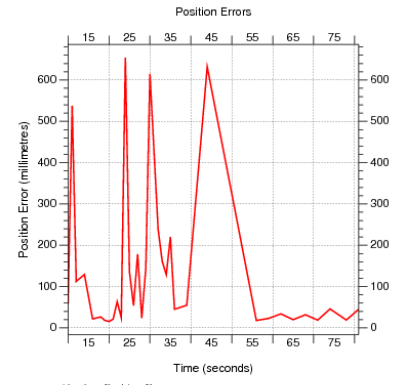

(a)

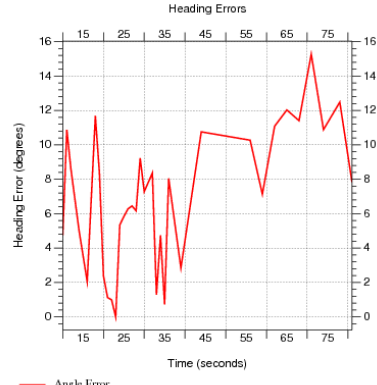

(b)
Fig. 12. (a) Position and (b) heading errors of the experiment in an relative large environment.

We have noticed that the robot should sense many observations from different locations to solve the multiple possibilities in the space, i.e., the robot is not able to estimate a unique possible location using only the fuzzy segments sensed from the initial position. In the sequence of Fig. 11(c), we can observe that; the robot does not know its initial location in the beginning and the robot's belief is distributed along the whole field, after some observations the robot's belief presents more than eight possible regions where it could be located, i.e., the localization method is not able to find out the robot's pose up to several seconds navigating. When the robot has traveled a distance, the ambiguity is solved and the real region of possibility is found. During the rest of the experiment, the localization method is able to maintain the robot's belief using the absolute and relative measurements. We also can observe that the real trajectory, which is shown in Fig. 11(a), is always inside the bounding box during the whole experiment.

The position and orientation errors are shown in Fig. 12. These errors are also calculated when the self-localization method estimates high reliability values, i.e., once the localization has converged. We also have noticed several peaks in the position error, even higher than the office-like experiment. As previously mentioned, these are due to the motion and its uncertainty model, which induces uncertainty in the robot's belief in all directions. The robot's motion is very unreliable and we are using a very rough motion model, the robot's belief increases much and if there is not enough absolute information to reduce this belief again, position error is increased. This problem is more critic in long corridors because the robot's belief is increased in all directions but the observations do not reduce the uncertainty in the direction of the corridor, and thus the belief is increased without bounds. Anyway, the results show that the position error is less than $65 \mathrm{~cm}$ during the experiment, but most of the time is less than $25 \mathrm{~cm}$. Concerning the heading error, we have not noticed any problem, and it is bounded in 16 degrees during the experiment, although the heading error is less than 7 degrees in average.

The computational cost is also evaluated. Besides the relative large environment, the fuzzy self-localization method allows processing the measures provided by the laser at an acceptable rate; in particular, the acting operation consumes 53 milliseconds for this environment, while the sensing operation consumes 17 milliseconds for each observation. Hence, given a perception of 5 fuzzy segments in average, the localization technique consumes 138 milliseconds in average using the same CPU than the other experiment.

\section{Conclusions}

This paper presents the integration of different techniques based on fuzzy logic for addressing the indoor localization problem using a wheeled robot with very unreliable odometry and equipped with a laser rangefinder. For perception, the fuzzy segment theory is used to maintain a coherent representation of the environment surrounding the robot, filtering out wrong detection and removing repetitious information by the integration of the readings of the laser rangefinder at different times. For self-localization, this coherent representation of the environment surrounding the robot is used to feed a fuzzy localization method based on a fuzzy grid.

The representation of the environment generated and maintained by the fuzzy segment theory is merely a local map of fuzzy segments. This representation consists on a set of fuzzy segments representing the bounds of the objects in the environment. In this work, we are only using walls to locate the robot, thus these fuzzy segments should to satisfy many conditions to filter out bounds of objects that probably are not walls and could induce wrong information into the localization method. The fuzzy segment method combines the information at segment level, which allows us to fuse the fuzzy segments generated at different times.

The self-localization method based on a fuzzy grid is fed by the local map of fuzzy segments generated by the perception system. This method presents the following advantages; i) it only needs an approximate sensor model, ii) it is able to represent the different aspects of uncertainty that affect to the measures, and iii) it is able to handle the ambiguity in the location avoiding to deal with the data association problem. Logically, the main disadvantage of this method is the fact that the environment is tessellated, and despite of the complexity is reduced using some simplifications, the computational cost is high in comparison to other techniques, especially local methods. This disadvantage is largely compensated by the stability of the method and its robustness, which are critical points in local methods with high uncertainty.

The whole system based on fuzzy logic has been experimentally validated in different environments. The experiments have proved that this approach is able to represent and deal with the uncertainty properly. In addition, the self-localization method is able to manage multi-hypotheses without addressing the data association problem, i.e., it is able to deal with the ambiguity and solve the global localization problem. In addition, the method is able to track the robot's location during the rest of the navigation.

\section{ACKNOWLEDGMENT}

This work has been supported by CICYT project DPI200407993-C03-02. The experimental part has been performed during a research period in the Mobile Robotics Laboratory of the Center for Applied Autonomous Sensor Systems (AASS) 
at the Department of Technology of Örebro University, Sweden. The authors would like to acknowledge Prof. Alessandro Saffioti for his supervision during this period.

\section{REFERENCES}

[1] I. Bloch and H. Maître. Fuzzy mathematical morphologies: a comparative study. Pattern Recognition, 28(9):1341-1387, 1995.

[2] I. Bloch and A. Saffiotti. Why robots should use fuzzy mathematical morphology. In Proc. of the 1st Int. ICSC-NAISO Congress on NeuroFuzzy Technologies, La Havana, Cuba, 2002.

[3] J. Borenstein, H. Everett, L. Feng and D. Wehe. Mobile Robot Positioning: Sensors and Techniques. Journal of Robotic Systems, Special Issue on Mobile Robots, 14(4):231-249, 1997.

[4] G.A. Borges and M.J. Aldon. Line Extraction in 2D Range Images for Mobile Robotics. Intelligent and Robotic Systems, 40(3):267-297, 2004

[5] W. Burgard, D. Fox, D. Henning and T. Schmidt. Estimating the Absolute Position of a Mobile Robot using Position Probability Grids. In American Association for Artificial Intelligence (AAAI), pages 896-901, 1996.

[6] P. Buschka, A. Saffiotti, and Z. Wasik. Fuzzy landmark-based localization for a legged robot. In Intelligent Robots and Systems (IROS), pages 12051210, Takamatsu, Japan, 2000.

[7] R.O. Duda and P.E. Hart. Pattern Classification and Scene Analysis. John Wiley and Sons, New York, 1973.

[8] J. Gasós and A. Martín. Mobile Robot Localization using Fuzzy Maps. In Fuzzy Logic in Artificial Intelligence, pages 207-224. 1995.

[9] J. Gasós and A. Martín. A Fuzzy Approach to Build Sonar Maps for Mobile Robots. Computers in Industry, 1(32):151-167, 1996.

[10] J. Gasós and A. Martín. Mobile Robot Localization using Fuzzy Maps. In Fuzzy Logic in Artificial Intelligence, LNAI 1188, pages 207-224. 1997.

[11] J. Gasós and A. Rosetti. Uncertainty Representation for Mobile Robots: Perception, Modeling and Navigation in Unknown Environments. Fuzzy Sets and Systems, 107(1):1-24, 1999.

[12] J. Gasós and A. Saffiotti. Integrating Fuzzy Geometric Maps and Topological Maps for Robot Navigation. In Proceedings of the 3rd International Conference on Soft Computing (SOCO), Genova, Italy, 1999.
[13] D. Fox, W. Burgard and S. Thrun. Markov Localization for Mobile Robots in Dynamic Environments. Journal of Artificial Intelligence Research, 11:391-427, 1999.

[14] D. Fox, J. Hightower, L. Liao, D. Schulz and G. Borriello. Bayesian Filtering for Location Estimation. IEEE Pervasive Comp. Mag., 2(3):2433, 2003.

[15] D. Herrero-Pérez, H. Martínez-Barberá and A. Saffiotti. Fuzzy SelfLocalization Using Natural Features in the Four-Legged League. In Proc. of the Int. RoboCup Symposium, Lisbon, pages 110-121, 2004.

[16] J. Leonard and H. Durrant-Whyte. Mobile robot localization by tracking geometric beacons. IEEE Transactions on Robotics and Automation, 7(3):376-382, 1991.

[17] G. Oriolo, M. Vendittelli, and G. Ulivi. Path planning for mobile robots via skeletons on fuzzy maps. Intelligent Automation and Soft Computing, 2(4):355-374, 1996.

[18] G. Oriolo, G. Ulivi and M. Venditelli. Real-Time Map Building and Navigation for Autonomous Mobile Robots in Unknow Environments. IEEE Transaction on Systems, Man and Cybernetics, 3(28):316-333, 1998.

[19] A. Saffiotti and L. Wesley. Perception-Based Self-Localization using Fuzzy Locations. In Workshop on Reasoning with Uncertainty in Robotics, pages 368-385, Amsterdam, NL, 1996.

[20] A. Saffiotti. The uses of fuzzy logic in autonomous robot navigation. Soft Computing, 1(4):180-197, 1997.

[21] V. Nguyen, A. Martinelli, N. Tomatis, and R. Siegwart. A Comparison of Line Extraction Algorithms using 2D Laser Rangefinder for Indoor Mobile Robotics. In IEEE/RSJ Intenational Conference on Intelligent Robots and Systems (IROS), Edmonton, Canada, 2005.

[22] K. Tanaka and M. Sugeno. Stability analysis and design of fuzzy control systems. Fuzzy Sets and Systems, 45:135-156, 1992.

[23] K. Tanaka and M. Sano. Trajectory stabilization of a model car via fuzzy control. Fuzzy Sets and Systems, 70:155-170, 1995.

[24] R. R. Yager. On a hierarchical structure for fuzzy modeling and control. IEEE Trans. on Systems, Man, and Cybernetics, 23(4):1189-1197, 1993.

[25] L.A. Zadeh. Fuzzy sets. Information and Control, 8:338-353, 1965.

[26] L.A. Zadeh. Fuzzy sets as a basis for a theory of possibility. Fuzzy Sets and Systems, (1):3-28, 1978.

[27] L. Zhang and B.K. Ghosh. Line Segment Based Map Building and Localization Using 2D Laser Rangefinder. In IEEE International Conference on Robotics and Automation, pages 2538-2543, San Francisco, CA, 2000. 